\title{
Consideration of the relationship between Kepler and cyclotron dynamics leading to prediction of a nonmagnetohydrodynamic gravity-driven Hamiltonian dynamo
}

\author{
P. M. Bellan \\ Applied Physics, Caltech, Pasadena, California 91125, USA
}

(Received 6 August 2007; accepted 30 October 2007; published online 10 December 2007)

\begin{abstract}
Conservation of canonical angular momentum shows that charged particles are typically constrained to stay within a poloidal Larmor radius of a poloidal magnetic flux surface. However, more detailed consideration shows that particles with a critical charge-to-mass ratio can have zero canonical angular momentum and thus can be both immune from centrifugal force and not constrained to stay in the vicinity of a specific flux surface. Suitably charged dust grains can have zero canonical angular momentum and in the presence of a gravitational field will spiral inwards across poloidal magnetic surfaces toward the central object and accumulate. This accumulation results in a gravitationally-driven dynamo, i.e., a mechanism for converting gravitational potential energy into a batterylike electric power source. (C) 2007 American Institute of Physics.
\end{abstract}

[DOI: $10.1063 / 1.2815791]$

\section{INTRODUCTION}

To the best of the author's knowledge, no plasma has ever been observed to make a Kepler orbit around the Sun, a planet, or a moon. For example, the Earth's magnetotail does not make Kepler orbits around the Earth nor does the solar wind make a Kepler orbit around the Sun. This is puzzling because in the astrophysical literature, plasmas are routinely presumed to make near-Kepler orbits in the presence of the gravitational field of a central object, e.g., accretion disks are modeled as magnetohydrodynamic (MHD) plasmas in a near-Kepler orbit around a central object. The qualifier "near" is used because it is conventionally presumed for gases and plasmas that a radial gradient of an isotropic pressure exists and that this pressure gradient provides a modest outward force that slightly reduces the amount of centrifugal force required to balance the inward force of gravity and so achieve a stable circular orbit (e.g., see Ref. 1 for a discussion of the implications of this effect in the context of a system consisting of gas and solid particles).

If Kepler orbiting plasmas are so ubiquitous in astrophysics, then why is there not even a single example in the great variety of plasma/gravitational situations in our own solar system? A Kepler orbit is essentially a property of a single point particle-it is not a property of a collection of independent point particles. For example, Earth and Mars are individually in Kepler orbits around the Sun, but the center of mass of Earth and Mars is not in a Kepler orbit around the Sun. If one replaced Earth and Mars by some statistically large number of point particles, then each could be in its own Kepler orbit around the Sun, but the center of mass of this configuration would not be in a Kepler orbit.

A collisional gas in a container could be in a Kepler orbit around the Sun because the walls of the container bind the particles to stay within a fixed distance of the center of mass so that the entire system can be considered as a point particle located at the center of mass. The transition from single point particle behavior to the behavior of a group of particles can be seen by considering a moon in close orbit around a massive planet. The tidal forces resulting from the gradient of the planet's gravitational force can be so strong as to overcome the binding forces and fracture the moon. The fragments would then follow distinct individual Kepler orbits, but the center of mass of these orbits would not follow a Kepler orbit. Similarly, in order for a gas (or plasma) to have its center of mass follow a Kepler orbit, there would have to be some binding force, such as would be provided by container walls, that would prevent the particle constituents of the gas (or plasma) from separating onto distinct Kepler orbits. Collisions might provide binding at the interior of a gas cloud, but not at the periphery because particles at the periphery moving away from the center would not encounter other particles with which they could collide. The periphery would simply expand into vacuum if there is no wall to prevent this expansion and the particles constituting the periphery would make Kepler orbits substantially different from the Kepler orbit calculated for the center of mass.

Accretion disks are composed of dust and gas and the dust-to-gas mass ratio is estimated ${ }^{2,3}$ to range from $\sim 10^{-2}$ to $\sim 1$. UV radiation photoionizes ${ }^{4-6}$ the dust and gas so the accretion disk can be considered as a dusty plasma ${ }^{7}$ consisting of charged dust grains, electrons, and ions. The magnetorotational instability ${ }^{8}$ (MRI) and the unipolar induction dynamo $^{9}$ (UID) assume accretion disks are axisymmetric ideal MHD plasmas and neglect dusty plasma physics effects. The MRI and UID additionally assume that accretion disks obey both Kepler dynamics and ideal MHD. Thus accretion disks are considered to be ideal MHD plasmas in a circular Kepler orbit about a central object; they are supposed to conserve angular momentum and have frozen-in magnetic flux. One can then ask what the trajectory of an individual particle in the accretion disk looks like. Since MHD is assumed and MHD is based on the assumption that all particles make cyclotron orbits, it seems that the indi- 
vidual particle in question should be making a cyclotron orbit. On the other hand, since the whole plasma is supposed to be making a circular Kepler orbit around the central object, then presumably this cyclotron-orbiting particle must also be making a Kepler orbit around the central object. A Keplerorbiting cyclotron orbit is not an obvious concept to visualize, at least to this author. This conceptual difficulty suggests that instead of assuming that a particle is simultaneously Kepler-orbiting and cyclotron orbiting, one should go back to first principles to investigate how Kepler and cyclotron orbits relate to each other. Perhaps it will then become obvious how to visualize a Kepler-cyclotron orbiting particle, or perhaps not.

\section{KEPLER EFFECTIVE POTENTIAL AND ORBITS}

Let us begin with a brief review of Kepler orbits. A particle $\sigma$ with conserved angular momentum $L_{\sigma}=m_{\sigma} r^{2} d \phi / d t$ has radial motion in the Kepler "effective" potential $^{10}$

$$
\chi_{\text {Kepler }}(r)=\frac{L_{\sigma}^{2}}{2 m_{\sigma} r^{2}}-\frac{m_{\sigma} M G}{r},
$$

where $M$ is the mass of a central object. Particles with energies at $\min \chi_{\text {Kepler }}(r)$ have circular trajectories with $r=L_{\sigma}^{2} / m_{\sigma}^{2} M G$, velocity $v_{\mathrm{K}}=\sqrt{M G / r}$, and constant angular velocity $\Omega=\sqrt{M G / r^{3}}$, whereas particles with energy exceeding $\min \chi_{\text {Kepler }}(r)$ have elliptical trajectories and variable angular velocity $d \phi / d t=L_{\sigma} / m_{\sigma} r^{2}{ }^{10}$

\section{GUIDING CENTER ORBITS IN COMBINED MAGNETIC AND GRAVITATIONAL FIELDS}

In contrast to Kepler dynamics, ${ }^{10}$ basic plasma theory ${ }^{11-13}$ shows that the guiding center of a charged particle in combined magnetic and gravitational fields, but no electric field, drifts at the velocity

$$
\mathbf{v}_{g \sigma}=\frac{m_{\sigma}}{q_{\sigma} B^{2}} \mathbf{g} \times \mathbf{B},
$$

where $\mathbf{g}$ is the gravitational acceleration. For a mass $M$ central object, $\mathbf{g}=M G \boldsymbol{\nabla}\left(r^{2}+z^{2}\right)^{-1 / 2}$, and so the guiding center drift in the $z=0$ plane is

$$
\mathbf{v}_{g \sigma}=\frac{M G}{r^{2} \omega_{c \sigma}} \hat{\phi}=\frac{\Omega}{\omega_{c \sigma}} v_{K} \hat{\phi},
$$

where $\omega_{c \sigma}=q_{\sigma} B_{z} / m_{\sigma}$ is the cyclotron frequency. It is seen that $\mathbf{v}_{g}$ is smaller than the Kepler velocity $v_{\mathrm{K}}$ by $\Omega /\left|\omega_{c \sigma}\right|$, an enormous ratio for electrons and ions since $\left|\omega_{c e}\right|$ and $\left|\omega_{c i}\right|$ are many orders of magnitude larger than $\Omega$ for typical field strengths. If all the particles move at a much slower velocity than the Kepler velocity, then how could the center of mass move at the Kepler velocity? Furthermore, the $\mathbf{v}_{g \sigma}$ add up to give the azimuthal current $\mathbf{J}_{g}=\sum n_{\sigma} q_{\sigma} \mathbf{v}_{g \sigma}=\rho \mathbf{g} \times \mathbf{B} / B^{2}$, where $\rho=\sum n_{\sigma} m_{\sigma}$. The gravitational drift current results mainly from heavy particle motion ${ }^{13}$ and gravity is balanced by the magnetic force (i.e., $\mathbf{J}_{g} \times \mathbf{B}=-\rho \mathbf{g}$ ), ${ }^{13}$ rather than by centrifugal force, which is insignificant for this example. The ideal MHD Ohm's law $\mathbf{E}+\mathbf{U} \times \mathbf{B}=0$ actually fails here, because the Hall term $\mathbf{J} \times \mathbf{B} /$ ne in the zero-pressure, generalized ${ }^{14}$ Ohm's law $\mathbf{E}+\mathbf{U} \times \mathbf{B}=\mathbf{J} \times \mathbf{B} /$ ne cannot be dropped since $\mathbf{J} /$ ne nearly equals the center-of-mass velocity $\mathbf{U}$.

What sort of trajectory does an actual charged particle follow in an astrophysical situation? Is it the Kepler orbit assumed in Refs. 8 and 9, or the much slower gravitational drift derived in Ref. 13? We show here that even though charged particles in a strong magnetic field can rotate at $v_{\mathrm{K}}$, as assumed in Refs. 8 and 9, the motion is not governed by Eq. (1), so charged particles (and hence a plasma) do not in general obey Kepler dynamics. This analysis leads to the realization that dust grains having a critical charge-to-mass ratio spiral in towards the central object and thus could provide a gravitationally powered dynamo suitable for driving astrophysical jets.

\section{HAMILTONIAN DESCRIPTION OF ORBITS IN COMBINED ELECTROMAGNETIC AND GRAVITATIONAL FIELDS}

We consider the axisymmetric charged particle Hamiltonian ${ }^{15-19}$

$$
\begin{aligned}
H= & \frac{m_{\sigma} v_{r}^{2}}{2}+\frac{m_{\sigma} v_{z}^{2}}{2}+\frac{1}{2 m_{\sigma} r^{2}}\left[P_{\phi}-\frac{q_{\sigma}}{2 \pi} \psi(r, z, t)\right]^{2} \\
& +q_{\sigma} V(r, z, t)-\frac{m_{\sigma} M G}{\sqrt{r^{2}+z^{2}}} .
\end{aligned}
$$

Here, $\psi=2 \pi r A_{\phi}$ is the poloidal flux and is related to the magnetic field by

$$
\mathbf{B}=-\frac{1}{2 \pi r} \frac{\partial \psi}{\partial z} \hat{r}-\frac{\partial A_{z}}{\partial r} \hat{\phi}+\frac{1}{2 \pi r} \frac{\partial \psi}{\partial r} \hat{z} .
$$

$V$ is the electrostatic potential, and we note that ideal MHD ultimately comes from approximations based on Eq. (4) and not Eq. (1). Because of axisymmetry, the particle's canonical angular momentum,

$$
P_{\phi}=m_{\sigma} r^{2} \frac{d \phi}{d t}+\frac{q_{\sigma}}{2 \pi} \psi(r, z, t)=L_{\sigma}+\frac{q_{\sigma}}{2 \pi} \psi(r, z, t),
$$

is invariant. ${ }^{16,17}$ Equation (4) is equivalent to the equation of motion

$$
m_{\sigma} \frac{d \mathbf{v}}{d t}=q_{\sigma}(\mathbf{E}+\mathbf{v} \times \mathbf{B})+m_{\sigma} \mathbf{g}
$$

with $\mathbf{E}=-\boldsymbol{\nabla} V-\partial \mathbf{A} / \partial t$.

We examine solutions to Eq. (7) in the $z=0$ plane for various charge-to-mass ratios, a uniform magnetic field $\mathbf{B}=B_{z} \hat{z}$, and two representative $V(r)$ profiles. In order to see the connection between the sense of particle injection and the magnetic field direction, the coordinate system definition we use here is such that positive $\phi$ is determined by the direction of injection of the particle; i.e., the particle always has initial positive $d \phi / d t$ by assumption. This definition means that $B_{z}$ could be positive or negative since the direction of the $z$ axis is determined by the sense of particle injection (i.e., we are defining the $z$ axis so that the particle is always injected in the counterclockwise direction) and not by the direction of B. Because $B_{z}$ can be positive or negative, $\omega_{c \sigma}$ will have the 
usual signage for $B_{z}>0$, but will have opposite polarity from convention if $B_{z}<0$. To see whether or not particles make Kepler orbits, we track particles starting with the same kinetic energy and $L_{\sigma}$ as a neutral particle undergoing an elliptical Kepler orbit. $a$ is defined to be the radius of the initial location and the $x$ axis lies in the direction from the central object to this location.

$L_{\sigma}$ is conserved if $q_{\sigma}=0$, but if $q_{\sigma} \neq 0$, then $P_{\phi}$ rather than $L_{\sigma}$ is the conserved quantity. ${ }^{16,17} B_{\phi}=0$ will be assumed (to be justified later). Time is normalized to the Kepler frequency of a neutral particle undergoing circular motion at $r=a$, i.e., to $\Omega_{0}=\sqrt{M G / a^{3}}$, and distances are normalized to $a$. The dimensionless variables are then $\bar{r}=r / a, \tau=\Omega_{0} t$, $\overline{\mathbf{v}}=\mathbf{v} / \Omega_{0} a, \quad \bar{L}=L_{\sigma} / m_{\sigma} a^{2} \Omega_{0}, \quad \bar{H}=H / m \Omega_{0}^{2} a^{2}, \quad \bar{V}(\vec{r})$ $=q_{\sigma} V(r) / m_{\sigma} \Omega_{0}^{2} a^{2}$, and using $\psi=B_{z} \pi r^{2}$,

$$
\bar{P}_{\phi}=\frac{P_{\phi}}{m_{\sigma} a^{2} \Omega}=\left(\frac{d \phi}{d \tau}+\frac{\omega_{c \sigma}}{2 \Omega_{0}}\right) \bar{r}^{2} .
$$

The dimensionless $\bar{z}=0$ plane Hamiltonian is thus

$$
\bar{H}=\frac{1}{2} \bar{v}_{r}^{2}+\frac{1}{2}\left(\frac{\bar{P}_{\phi}}{\bar{r}}-\frac{\omega_{c \sigma}}{2 \Omega_{0}} \bar{r}\right)^{2}+\bar{V}(\bar{r})-\frac{1}{\bar{r}} .
$$

Rotation of a plasma in a magnetic field polarizes the plasma radially, and the resulting $\bar{V}(\vec{r})$ corresponds to the voltage to which the plasma capacitor is charged. ${ }^{17}$ There is thus no natural $\bar{V}(\vec{r})$ profile and hence no natural rotational velocity (e.g., see Refs. 17 and 20). We consider two representative cases: (i) $\bar{V}(r)=0$ and (ii) $\bar{V}(r)=2 \bar{r}^{1 / 2} \omega_{c \sigma} / \Omega_{0}$. Case (i) corresponds to Eq. (2), while case (ii) corresponds to the "Kepler" equilibria assumed in Refs. 8 and 9. A third possibility, not discussed here (see Ref. 21), sets $\bar{V}(\bar{r})$ to give a rotation velocity equal to that of the central object (so-called "corotational velocity").

We consider all possible values of $\omega_{c \sigma} / \Omega_{0}$, namely, $\left|\omega_{c \sigma} / \Omega_{0}\right| \ll 1,\left|\omega_{c \sigma} / \Omega_{0}\right| \gg 1$, and $\left|\omega_{c \sigma} / \Omega_{0}\right|=\mathcal{O}(1)$, with $\omega_{c \sigma} / \Omega_{0}$ either positive or negative. $\left|\omega_{c \sigma} / \Omega_{0}\right| \gg 1$ is typical for electrons, ions, and large charge-to-mass ratio dust grains, whereas $\left|\omega_{c \sigma} / \Omega_{0}\right| \ll 1$ corresponds to dust grains with very small charge-to-mass ratios or macroscopic charged particles such as spacecraft. ${ }^{22}$ The voltage $V_{d}$ to which a dust grain becomes charged depends on the charging mechanism and the dust grain size; $V_{d}$ typically lies in the range $1 \mathrm{~V}<\left|V_{d}\right|<100 \mathrm{~V}$. Since the dust grain charge is $Q_{d}=4 \pi \varepsilon_{0} r_{d} V_{d}$, the charge-to-mass ratio $Q_{d} / m_{d}=3 \varepsilon_{0} V_{d} / r_{d}^{2} \rho_{d}$ lies in the range $10^{-4}-10^{2} \mathrm{C} / \mathrm{kg}$ for typical dust grain radii $0.1 \mu \mathrm{m}<r_{d}<10 \mu \mathrm{m}$ and typical dust grain intrinsic mass density $\rho_{d}=10^{3} \mathrm{~kg} \mathrm{~m}^{-3}$. The dust grain $\omega_{c d} / \Omega$ ratio is thus 9-15 orders of magnitude smaller than that of an electron and 6-12 orders of magnitude smaller than that of an ion.

The last two terms in Eq. (9) can be written as a normalized effective potential,

$$
\bar{\chi}(\bar{r})=\frac{1}{2}\left(\frac{\bar{P}_{\phi}}{\bar{r}}-\frac{\omega_{c \sigma}}{2 \Omega_{0}} \bar{r}\right)^{2}+\bar{V}(\bar{r})-\frac{1}{\bar{r}} .
$$

If $q_{\sigma}=0$, then $\omega_{c \sigma}=0, \bar{V}=0$ and $\bar{\chi} \rightarrow \bar{\chi}_{\text {Kepler }}$, in which case Kepler dynamics ${ }^{10}$ is retrieved.
However, when $\omega_{c \sigma} \neq 0$ and $\bar{V}(\bar{r})$ is arbitrary, the dynamics is non-Keplerian, and Eq. (10) has minima when

$$
\left(\frac{\bar{P}_{\phi}}{\bar{r}^{2}}\right)^{2}-\left(\frac{\omega_{c \sigma}}{2 \Omega_{0}}\right)^{2}-\frac{1}{\bar{r}} \frac{\partial \bar{V}}{\partial \bar{r}}-\frac{1}{\bar{r}^{3}}=0 .
$$

By using Eq. (8), Eq. (11) can be recast as

$$
\left(\frac{d \phi}{d \tau}\right)^{2}+\frac{\omega_{c \sigma}}{\Omega_{0}} \frac{d \phi}{d \tau}-\frac{1}{\bar{r}} \frac{\partial \bar{V}}{\partial \bar{r}}-\frac{1}{\bar{r}^{3}}=0
$$

so a particle with $\bar{H}$ equal to the effective potential minimum has an angular velocity

$$
\frac{d \phi}{d \tau}=-\frac{\omega_{c \sigma}}{2 \Omega_{0}} \pm \sqrt{\left(\frac{\omega_{c \sigma}}{2 \Omega_{0}}\right)^{2}+\frac{1}{\bar{r}} \frac{\partial \bar{V}}{\partial \bar{r}}+\frac{1}{\bar{r}^{3}}} .
$$

Figure 1 plots $\chi(\vec{r})$ and numerically calculated $x-y$ plane trajectories for a range of $\omega_{c \sigma} / \Omega_{0}$ values and for the two $\bar{V}(\bar{r})$ cases. In all trajectory calculations, the particle initial position is $\bar{x}=1, \bar{y}=0$, and the initial velocity is $\bar{v}_{x}=0.4, \bar{v}_{y}=1$ (i.e., particles start at the same position with the same initial velocity and the same initial mechanical angular momentum $\left.L_{\sigma}\right)$. The trajectories are calculated from $\tau=0$ to $4 \pi$ (i.e., two circular Kepler orbit periods) and the energy $\bar{H}$ is shown as a dashed line in the effective potential plots. An $\bar{r}=1$ reference circle (dashed) is shown in the trajectory plots.

Figure 1 shows that the trajectory depends strongly on both the $\bar{V}(\vec{r})$ profile and on $\omega_{c \sigma} / \Omega_{0}$. The $\omega_{c \sigma} / \Omega_{0}=0$ situation (fifth row of Fig. 1) is a classic elliptical Kepler orbit as prescribed by Eq. (1) and is independent of $\bar{V}(\bar{r})$ because a neutral particle is insensitive to electromagnetic fields. However, when $\omega_{c \sigma} / \Omega_{0}$ is finite, Fig. 1 shows that the effective potential and trajectories differ qualitatively from the classic neutral particle effective potential and elliptical Kepler orbit. It is therefore incorrect to characterize a plasma composed of particles with $\left|\omega_{c \sigma} / \Omega_{0}\right| \gg 1$ as being in a Kepler orbit (as done in the MRI and UID models) because particles in such a plasma are not governed by Eq. (1) and, for example, do not make elliptical orbits with the central object at one focus of the ellipse (such orbits are the "hallmark" of Kepler dynamics).

Insight into the orbits shown in Fig. 1 can be obtained by examining solutions to Eq. (13). For $\left|\omega_{c \sigma} / 2 \Omega_{0}\right| \ll 1$ and $\bar{V}(\bar{r})=0$, Eq. (13) has the roots

$$
\frac{d \phi}{d \tau}= \pm \frac{1}{\bar{r}^{3 / 2}}-\frac{\omega_{c \sigma}}{2 \Omega_{0}}
$$

so heavy charged dust grains with $\bar{H}$ equal to the minimum of the effective potential make circular orbits with a small $\omega_{c \sigma} / 2 \Omega_{0}$ correction to the Kepler frequency. Heavy charged dust grains with $\bar{H}$ slightly above this minimum will make 


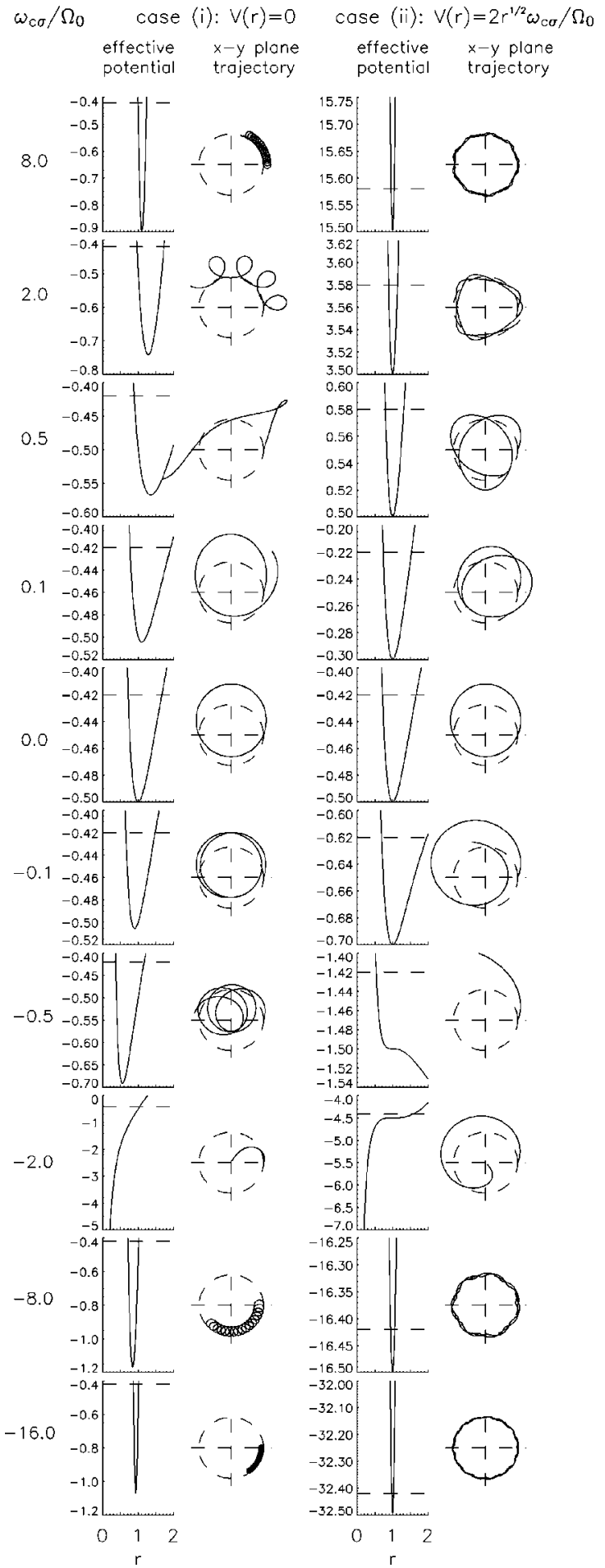

FIG. 1. Effective potentials and plane trajectories for particles starting at $\bar{x}=1, \bar{y}=0$, with initial velocity $\bar{v}_{x}=0.4, \bar{v}_{y}=1$, with a range of $\omega_{c \sigma} / \Omega_{0}$ values and two $\bar{V}(\bar{r})$ cases. Elliptical Kepler orbits [i.e., Eq. (1) effective potential] occur only when $\omega_{c \sigma} / \Omega=0$. Particles with $\omega_{c \sigma} / \Omega_{0}=-2$ fall towards $\bar{r}=0$ and have $\bar{P}_{\phi}=0$. No particles make Kepler-like elliptical orbits when $\left|\omega_{c \sigma} / \Omega_{0}\right| \gg 1$.

precessing elliptical Kepler orbits having small $\omega_{c \sigma} / 2 \Omega_{0}$ corrections (see $\omega_{c \sigma} / \Omega_{0}= \pm 0.1$ cases in Fig. 1) and these corrections will increase with the charge-to-mass ratio.

For $\left|\omega_{c \sigma} / 2 \Omega_{0}\right| \gg 1$ and $\bar{V}(\bar{r})=0$, the two roots of Eq. (13) are

$$
\frac{d \phi}{d \tau}=-\frac{\omega_{c \sigma}}{\Omega_{0}}, \quad \frac{d \phi}{d \tau}=\frac{1}{\bar{r}^{3}} \frac{\Omega_{0}}{\omega_{c \sigma}} .
$$

The first root corresponds to a so-called axis-encircling cyclotron orbit; ${ }^{16}$ this root is not likely to be physically realizable in astrophysical situations since its corresponding azimuthal velocity exceeds the Kepler velocity by the large ratio $\left|\omega_{c \sigma} / \Omega_{0}\right|$. Normal cyclotron orbits correspond to a particle oscillating ${ }^{16,17}$ in $r$ about a local minimum of $\chi(r)$ and are associated with the second root in Eq. (15). The second root is just $\mathbf{v}_{g}$ prescribed by Eq. (2) (see Refs. 11-13) and, as discussed above, is smaller than $\mathbf{v}_{\mathrm{K}}$ by the factor $\left|\Omega_{0} / \omega_{c \sigma}\right|$. In reality, $\left|\Omega_{0} / \omega_{c \sigma}\right|$ would be so enormous that electrons and ions would have negligible azimuthal displacement during one Kepler period of a neutral particle. These slow drift orbits are shown in the top and bottom rows of case (i) in Fig. 1. In accordance with Eq. (2), heavy particles drift faster, negative and positive particles drift in opposite directions, and the drift velocity decreases as $B_{z}$ increases.

For case (ii), $\bar{V}(\bar{r})=2 \bar{r}^{1 / 2} \omega_{c \sigma} / \Omega$, and thus Eq. (12) becomes

$$
\left(\frac{d \phi}{d \tau}-\frac{1}{\bar{r}^{3 / 2}}\right)\left(\frac{d \phi}{d \tau}+\frac{1}{\bar{r}^{3 / 2}}+\frac{\omega_{c \sigma}}{\Omega_{0}}\right)=0,
$$

where one root is the circular Kepler-like orbit $d \phi / d \tau=1$ with $\bar{r}=1$. Although the $d \phi / d \tau=1$ root looks superficially like a neutral particle Kepler orbit, the orbits are not elliptical, but nearly circular, and, as in tokamaks and spheromaks, stay within a poloidal Larmor orbit of a constant $\psi$ surface. $^{17,23}$ The effective potential minimum has the same radial location as Eq. (1) but the profile is an extremely narrow trough with a large vertical offset (positive or negative, depending on the charge polarity), not a shallow broad well as for Eq. (1).

\section{ORBITS OF PARTICLES WITH ZERO CANONICAL ANGULAR MOMENTUM: DYNAMO FOR DRIVING ASTROPHYSICAL JETS}

A strange behavior is evident in the $\omega_{c \sigma} / \Omega_{0}=-2.0$ row of Fig. 1: The effective potential goes to minus infinity on the left and the particle spirals inwards toward the origin in the $x-y$ trajectory plots. This corresponds to $P_{\phi}=0$ and is unlikely for electrons or ions because they typically have $\left|\omega_{c \sigma} / \Omega_{0}\right| \gg 1$. However, $P_{\phi}=0$ could occur for dust grains because, being heavy, dust grains have $\left|\omega_{c \sigma} / 2 \Omega_{0}\right|$ many orders of magnitude smaller than electrons or ions. As seen from Eq. (8), $\bar{P}_{\phi}=0$ occurs if $d \phi / d \tau=-\omega_{c \sigma} / 2 \Omega_{0}$ or, in unnormalized quantities $P_{\phi}=0$ occurs when $d \phi / d t=-\omega_{c \sigma} / 2$, in which case $d \phi / d t$ also becomes invariant. In this situation the normalized effective potential, Eq. (10), reduces to

$$
\bar{\chi}(\bar{r})=\frac{1}{8}\left(\frac{\omega_{c \sigma}}{\Omega_{0}}\right)^{2} \bar{r}^{2}+\bar{V}(\bar{r})-\frac{1}{\bar{r}},
$$

which has the remarkable feature that no centrifugal repulsion exists so the particle always falls towards $\bar{r}=0$ with constant $d \phi / d \tau$ (i.e., it spirals in). This differs qualitatively from $\bar{P}_{\phi} \neq 0$ particles which are constrained to orbit at a 
fixed average radius. The normalized radial force acting on a $\bar{P}_{\phi}=0$ particle is

$$
\bar{F}=-\frac{\partial \bar{\chi}}{\partial \bar{r}}=-\frac{1}{4}\left(\frac{\omega_{c \sigma}}{\Omega_{0}}\right)^{2} \bar{r}-\frac{\partial \bar{V}}{\partial \bar{r}}-\frac{1}{\bar{r}^{2}},
$$

which is negative for any potential having $\partial \bar{V} / \partial \bar{r} \geq 0$. Thus, $\bar{F}$ is negative for both the $\bar{V}=0$ and the "Kepler" potential $\bar{V}(\bar{r})=2 \bar{r}^{1 / 2} \omega_{c \sigma} / \Omega$. If there is a distribution of dust grain sizes, then a corresponding distribution of $\omega_{c \sigma} / \Omega_{0}$ values will develop and some subset will have $\omega_{c \sigma} / \Omega_{0}=-2$. The situation $\omega_{c \sigma} / \Omega_{0}=-2$ could occur for negatively charged dust grains injected with $B_{z}>0$ or for positively charged dust grains with $B_{z}<0$. This latter case is the likely one, and if one were to adopt the common convention that the $z$ axis is defined by the direction of $\mathbf{B}$, this would correspond to retrograde injection.

Dust grains accreting to a circumstellar disk will typically absorb stellar UV photons ${ }^{4}$ and become positively charged by emitting photoelectrons. ${ }^{6,7}$ Some of these dust grains will satisfy $d \phi / d t=-\omega_{c \sigma} / 2$ if $B_{z}<0$ and thus have $\bar{P}_{\phi}=P_{\phi}=0$. These dust grains have mechanical angular momentum $L_{\sigma}=m_{\sigma} r^{2} d \phi / d t$ at the instant before becoming charged by photoemitting electrons; i.e., they have mechanical angular momentum $L_{\sigma}=-q_{\sigma} \psi / 2 \pi$ at the instant before they become charged. Since neither $r$ nor $d \phi / d t$ is changed at the instant of charging, their canonical angular momentum $P_{\phi}=L_{\sigma}+q \psi / 2 \pi$ becomes zero at the instant after charging.

The infalling $\bar{P}_{\phi}=0$ dust grains will accumulate at small $\bar{r}$ and create a positive space charge there. The photoemitted electrons, stranded at large $\bar{r}$ (since electrons have $P_{\phi} \neq 0$ ), will create a corresponding negative space charge at large $\bar{r}$. The positive and negative space charges will tend to cancel any polarization charge; e.g., the polarization charge associated with an initial $\bar{V}(\bar{r})=2 \bar{r}^{1 / 2} \omega_{c \sigma} / \Omega$ potential. Accumulation of infalling $\bar{P}_{\phi}=0$ positive dust grains will eventually create an outward radial electric field $E_{r}^{*}$ [i.e., opposite direction to that associated with the $\bar{V}(\vec{r})=2 \bar{r}^{1 / 2} \omega_{c \sigma} / \Omega$ potential]. This accumulation will cease when $E_{r}^{*}$ becomes sufficiently large to create a force $q_{\sigma} E_{r}^{*}$, which cancels $F$. In un-normalized variables, this cancellation occurs when $E_{r}^{*}=\partial V_{\text {other }} / \partial r+\left(r \omega_{c \sigma}^{2} / 4+M G / r^{2}\right) m_{\sigma} / q_{\sigma}$, where $V_{\text {other }}$ is the potential profile that would exist due to particles other than the accumulating $P_{\phi}=0$ dust grains. The inward falling $P_{\phi}=0$ dust grains constitute a radially inward conduction current, so $\mathbf{J} \cdot \mathbf{E}$ is negative and the system converts gravitational potential energy into available electrical power; i.e., it is a dynamo. The positive voltage near $r=0$ will drive bipolar axial electric currents $I$ outwards from the $z=0$ plane. These currents will deplete the positive space charge, which will result in a net force $F-q_{d} E_{r}^{*}$, which will drive additional $\bar{P}_{\phi}=0$ dust grains towards $r=0$, where they will replenish the positive space charge. Thus, the system continuously converts the gravitational potential energy of the $P_{\phi}=0$ accreting dust grains into a batterylike electrostatic potential that drives the poloidal current of an astrophysical jet. The jet itself is accelerated by the $\partial B_{\phi}^{2} / \partial z$ force $^{24-26}$ associated with the axial nonuniformity of the jet poloidal current $I(r, z)$ since $B_{\phi}(r, z)=\mu_{0} I(r, z) / 2 \pi r$. The antisymmetry of $I$ with respect to $z$ means that $B_{\phi}=0$ at $z=0$, consistent with the assumption made earlier.

We note that the ability of $P_{\phi}=0$ particles to cross magnetic flux surfaces has recently been observed in a laboratory experiment. $^{27}$

The assumption used in this paper that $B_{z}$ is spatially uniform is a simplifying idealization that enables the analysis to be both brief and focused on the distinction between Kepler and charged particle orbits. However, an actual accretion disk will almost certainly have $B_{z}$ depend on both $r$ and on $z$, so in order to satisfy $\boldsymbol{\nabla} \cdot \mathbf{B}=0$, there will also have to be a $B_{r}(r, z)$. This indicates that the axisymmetric magnetic field would be best described using a poloidal flux function $\psi(r, z)$; i.e., $\mathbf{B}(r, z)=(2 \pi)^{-1}\left[\nabla \psi \times \nabla \phi+\mu_{0} I(r, z) \nabla \phi\right]$. This more general description of the magnetic field has been used in Ref. 28, a much lengthier analysis, where threedimensional particle orbits in an approximately selfconsistent magnetic field are considered using a generalization of the Hamiltonian method presented here. Specifically, the poloidal flux function $\psi(r, z)$ in Ref. 28 results from a toroidal current due to toroidal motion of charged particles, and particles are not restricted to the $z=0$ plane, as in the present paper.

The dust grains might be so densely packed as to be optically thick, in which case photons from the central object would not reach the dust and the dust would not become charged; this would constitute a so-called "dead-zone." The condition for a dust cloud to be optically thick is $n \sigma L>1$, where $n$ is the dust density, $\sigma$ is the dust cross section, and $L$ is the characteristic length scale of the dust cloud. However, the condition for a dust cloud to be collisional is also $n \sigma L>1$, and thus dust grains in an optically thick cloud would be collisional. This collisional, optically thick state would likely be transient, because collisions are expected to cause coagulation of the dust grains, ${ }^{1}$ in which case their radius $r_{d}$ will increase. Since the mass of an individual dust grain is $m_{d}=4 \pi \rho_{d} r_{d}^{3} / 3$ and since coagulation does not change the total mass $M$ of all the dust grains, the number $N=M / m$ of dust grains and hence the density $n \sim N / L^{3}$ of dust grains scales as $r_{d}^{-3}$. Because the dust grain cross section $\sigma$ scales as $r_{d}^{2}$, the product $n \sigma$ scales as $r_{d}^{-1}$, and so $n \sigma$ decreases as a result of coagulation. Coagulation of dust grains will thus reduce $n \sigma$ until $n \sigma L$ becomes less than unity, in which case the dust cloud will become collisionless and optically thin. At this point, dust would become charged (ionized) via photoemission and commence the collisionless trajectories discussed here. The dead zones would thus disappear as a result of coagulation. This issue is discussed in more detail in Ref. 28.

Reference 28 discusses several other issues, including the charging rate of dust grains (i.e., effective ionization rate of dust grains), collisions of dust grains with gas and other dust grains, and the topological properties of astrophysical jets. These various issues are used to define a parameter space for astrophysical jets powered by the gravitational energy released by accreting $P_{\phi}=0$ dust grains. A selfconsistent set of parameters is given for the example of the 
circumstellar accretion disk of a young stellar object (YSO).

${ }^{1}$ S. J. Weidenchilling, Space Sci. Rev. 92, 295 (2000).

${ }^{2}$ J. S. Greaves, V. Mannings, and W. S. Holland, Icarus 143, 155 (2000).

${ }^{3}$ J. C. Augereau, A. M. Lagrange, D. Mouillet, J. C. B. Papaloizou, and P.

A. Grorod, Astron. Astrophys. 348, 557 (1999).

${ }^{4}$ S. Wolf, Astrophys. J. 582, 859 (2003).

${ }^{5}$ P. Lee, Icarus 124, 181 (1996).

${ }^{6}$ A. A. Sickafoose, J. E. Colwell, M. Horanyi, and S. Robertson, Phys. Rev. Lett. 84, 6034 (2000).

${ }^{7}$ F. Verheest, Waves in Dusty Space Plasmas (Kluwer, Dordrecht, 2000).

${ }^{8}$ S. A. Balbus and J. F. Hawley, Astrophys. J. 376, 214 (1991); Rev. Mod. Phys. 70, 1 (1998).

${ }^{9}$ R. V. E. Lovelace, Nature (London) 262, 649 (1976); A. G. Bell and S. G. Lucek, Mon. Not. R. Astron. Soc. 277, 1327 (1995).

${ }^{10}$ L. D. Landau and E. M. Lifshitz, Mechanics (Pergamon, Oxford, 1960), p. 35.

${ }^{11}$ H. Alfvén, Cosmical Electrodynamics (Clarendon, Oxford, 1950), p. 15.

${ }^{12}$ L. Spitzer, Physics of Fully Ionized Gases (Interscience, New York), p. 5.

${ }^{13}$ P. A. Sturrock, Plasma Physics (Cambridge University Press, Cambridge, 1994), pp. 24-25.

${ }^{14} \mathrm{M}$. Goosens, An Introduction to Plasma Astrophysics and Magnetohydro- dynamics (Kluwer, Dordrecht, 2003), p. 94.

${ }^{15}$ C. Störmer, The Polar Aurora (Clarendon, Oxford, 1955).

${ }^{16} \mathrm{G}$. Schmidt, Physics of High Temperature Plasmas (Academic, New York, 1979), p. 34.

${ }^{17}$ B. Lehnert, Nucl. Fusion 11, 485 (1971).

${ }^{18}$ H. R. Dullin, M. Horanyi, and J. E. Howard, Physica D 171, 178 (2002).

${ }^{19}$ J. V. Shebalin, Phys. Plasmas 11, 3472 (2004).

${ }^{20}$ J. Ghosh, R. C. Elton, H. R. Griem, A. Case, R. Ellis, A. B. Hassam, S. Messer, and C. Teodorescu, Phys. Plasmas 11, 3813 (2004).

${ }^{21}$ J. E. Howard, M. Horanyi, and G. R. Stewart, Phys. Rev. Lett. 83, 3993 (1999).

${ }^{22}$ H. B. Garrett and A. C. Whittlesey, IEEE Trans. Plasma Sci. 28, 2017 (2000).

${ }^{23}$ This is well known in toroidal confinement devices, a typical proof is given in P. M. Bellan, Spheromaks (Imperial College Press, London, 2000), pp. 207-208.

${ }^{24}$ P. M. Bellan, Phys. Rev. Lett. 69, 3515 (1992).

${ }^{25}$ P. M. Bellan, Phys. Plasmas 10, 1999 (2003).

${ }^{26}$ S. You, G. S. Yun, and P. M. Bellan, Phys. Rev. Lett. 95, 045002 (2005).

${ }^{27}$ S. K. P. Tripathi, P. M. Bellan, and G. S. Yun, Phys. Rev. Lett. 98, 135002 (2007).

${ }^{28}$ P. M. Bellan, Astrophys. J. (submitted). 